\title{
Competitividad de las organizaciones productoras de cacao (Theobroma cacao I) en el sureste de Nicaragua
}

\author{
Norman Javier Saballos ${ }^{1}$ \\ Carlos Manuel Amador ${ }^{2}$ \\ Wilson Antonio Calero Borge ${ }^{3}$
}

\section{Resumen}

Se identificó los factores relacionados a la competitividad de las organizaciones de productores que comercializaron cacao durante el 2014, en la región sureste de Nicaragua. Para identificar los factores relacionados a la competitividad, se estudiaron a través de la "Cadena de Valor" cada una de las actividades primarias y de apoyo, así los indicadores de la competitividad; la productividad, calidad del producto, costos, cuota de mercado, permanencia en el mercado y la rentabilidad. El estudio se realizó mediante encuestas a representantes de siete organizaciones.

Palabras clave: Cadena de valor; ventajas comparativas; estrategias competitivas.

\section{Summary}

The factors related to the competitiveness of the producer organizations that commercialized cacao during 2014 were identified in the southeastern region of Nicaragua. To identify the factors related to competitiveness, each of the primary and support activities were studied through the "Value Chain", as well as the indicators of competitiveness, productivity, product quality, costs, market share, market permanence and profitability. The study was conducted through surveys of representatives of seven organizations.

Key Words: Value chain; Comparative advantages; Competitive Strategies.

\section{Introducción}

La competencia entre las empresas ya no es entre productos, sino entre sistemas productivos, en especial entre modelos de integración. La idea de centrar el estudio en las empresas de pequeños productores en el sureste de Nicaragua, se debe a la importancia que tienen estas organizaciones en la comercialización de cacao, y por

1 Master en Agronegocios. Coordinador de la Facultad de Agroforestal de la Bluefields Indian and Caribbean University - El Rama. Correo: saballosnj@gmail.com

2 Master en Agronegocios. Consultor senior. Correo: cammador@gmail.com

3 Master en Agroforestería Tropical. Coordinador de Investigación de la Universidad de las Regiones Autónomas de la Costa Caribe Nicaragüense - Recinto Universitario Nueva Guinea. Correo: investigación.ng@uraccan.edu.ni

Recibido: 27/07/2017 Aprobado: 23/11/2017

Saballos, N., J., Amador, C., \& Calero, W., A. (2017). Competitividad de las organizaciones productoras de cacao (Theobroma cacao I) en el sureste de Nicaragua. Ciencia E Interculturalidad, 21(2), 104-114. 
la escasez de datos relacionados al análisis de la gestión y competitividad de las organizaciones perteneciente al sector cacao.

Como primer peldaño hacia el logro de la competitividad, según Porter (2006), hay que reconocer en las cadenas de valor que los proveedores, compradores y canales son aliados en la competencia y no sólo componentes de una transacción. En este contexto, la cadena de valor permite dividir a la empresa en sus actividades estratégicamente relevantes a fin de entender el comportamiento de los costos (Porter, 2006).

Un análisis integral de la competitividad de las organizaciones de productores de cacao en la región sureste de Nicaragua, y análisis mediante la herramienta cadena de valor, muestra que cada organización difiere notablemente en funcionamiento productivo y empresarial. Por lo anterior, este estudio se planteó identificar los principales puntos críticos que frenan la competitividad de las organizaciones y las actividades más frecuentes en la producción de cacao que generan ventajas competitivas y medir los factores relacionados con la competitividad, como insumo para un posterior planteamiento de estrategias de consolidación de ventajas competitivas.

Los resultados del estudio indican que la competitividad de las organizaciones cacaoteras de la región sureste de Nicaragua es demasiado baja. Se concluye, que los factores más importantes que influyen en la competitividad de las organizaciones cacaoteras son los siguientes: bajos volúmenes de cacao comercializados, las organizaciones aportaron un 2.4\% al volumen que exportó el país durante el 2014; baja calidad del producto comercializado, un $35 \%$ de la producción no cumplió los estándares de calidad que exige el mercado internacional, y falta de integración de tecnologías al proceso productivo. En este sentido la región sureste de Nicaragua tiene poca participación en el mercado internacional del cacao y las organizaciones cacaoteras no son muy competitivas.

\section{Revisión de literatura}

\section{Cadena de valor}

Las organizaciones se alimentan del entorno, la industria y de sí mismas para su generación y propuesta de valor. Un modelo para desarrollar dicha proposición es la cadena de valor. Porter (1987) afirma que la empresa es un conjunto de actividades cuyo fin es diseñar, fabricar, comercializar, entregar y apoyar un producto. Se puede representarla por medio de la cadena de valor. En esta línea, la cadena de valor "desagrega a la empresa en una cadena de actividades secuenciales" (Grant 1996: 167). 


\section{La cadena de valor de McKinsey \& Company}

En 1980, McKinsey \& Company plantea un modelo que buscó desagregar a la empresa en una cadena de actividades secuenciales construida sobre el concepto de «sistema de negocios» (Guitart, 2005). Es un concepto que pone en evidencia el hecho de que todos los negocios son una cadena de actividades que van desde la entrada de insumos, materias primas, entre otros, por medio de compras u otros procesos, hasta el servicio postventa. Cada eslabón debe desempeñar un papel en la contribución de la explotación de las fuentes potenciales de ventajas competitivas sobre los competidores (Manso, 2003). Este planteamiento es el primigenio en el desarrollo del concepto de la cadena de valor y de los modelos relacionados con ella.

\section{Cadena de valor de Michael E. Porter}

Porter (1985), define la cadena de valor como: "la herramienta básica para analizar las fuentes de la ventaja competitiva de una empresa, ya que examina todas las actividades que realizan la empresa y su manera de interactuar. No se puede entender la ventaja competitiva de una empresa si no se examina la empresa en su conjunto, ya que la ventaja nace de cada una de las actividades que la empresa ejecuta al diseñar, fabricar, comercializar, entregar y apoyar su producto".

\section{Competitividad}

Müller (1995), distingue los términos de competencia y competitividad. Por el primero, se puede entender como parte de la lucha económica, y la capacidad para la competencia que desemboca en la rivalidad entre grupos de vendedores.

Para Ruesga y Da Silva (2007), la competitividad a nivel de empresas se define como la capacidad de las mismas para competir en los mercados, ganar parcelas de mercado, aumentar sus beneficios y crecer. En este sentido, los indicadores de competitividad más utilizados son: la cuota de mercado, los beneficios, los dividendos y el volumen de inversiones.

\section{Materiales y métodos}

\section{Diseño y población de estudio}

Se realizó una investigación cuantitativa con un alcance correlacional causal, utilizando el "diseño no experimental transversal". Para el estudio se utilizaron datos de todo un año, del 1 de enero al 31 de diciembre del 2014. Se trabajó con los datos de siete organizaciones de productores de cacao de la región sureste de Nicaragua, que tuvieron participación en el mercado nacional e internacional del cacao en el 2014. 


\section{Recolección de datos y descripción de las variables}

En la recolección de datos se utilizaron técnicas de revisión documental, observación directa y encuestas. Se realizó la revisión documental sobre mapeos previos realizados en la zona, y la revisión de documentación interna de las organizaciones, exceptuando la información financiera. La observación directa fue realizada en los centros de beneficiados de postcosecha; fermento, presecado, secado, bodega y procesamiento de productos, a fin de comprender la realidad cotidiana de las organizaciones y conocer los procesos del beneficiado de cacao y observar los logros significativos obtenidos en relación con la calidad del producto ofertado.

Se aplicó el cuestionario a los presidentes, responsables administrativos, responsables de comercialización y responsables de calidad de cada organización. En el cuestionario se consideran dos tipos de variables; cadena de valor y competitividad. Se tabuló la información obtenida en el programa Microsoft Office Excel 2013.

\section{Análisis estadístico}

Se utilizó el coeficiente de correlación de Pearson ( $\mathrm{r}$ y r2) para la comprobación de la hipótesis de trabajo, y por último se realizó la interpretación de los resultados descriptivos.

\section{Para comprobar la hipótesis, se consideraron los pasos siguientes:}

1. Elaboración de un diagrama de dispersión, con los datos obtenidos del análisis de los indicadores que componen la cadena de valor; para verificar que los datos obtenidos no estuvieran dispersos, se eliminaron los valores que afectaban de forma negativa las relaciones entre variables, se encontraron dos indicadores que fueron eliminados, quedando al final solo siete indicadores válidos.

2. Se realizó la Correlación de Pearson, para la relación entre cadena de valor y competitividad; para determinar el grado de relación de las variables y el porcentaje de explicación entre los mismos (r y r2). La Correlación de Pearson se realizó con la ayuda del programa estadístico Infostat (2015). Con base en lo anterior, se pudo interpretar el grado de asociación entre las variables de investigación

\section{Resultados y discusión}

Durante el 2014, el área cultivada de cacao en la región estuvo en el orden de las 906.03 ha-1, de las cuales el 59\% estaban en producción, obteniéndose una producción anual de 84.72 toneladas de cacao seco, estos datos reflejan un bajo rendimiento del cultivo $158.5 \mathrm{~kg}$ ha-1 año-1 cacao seco. Este rendimiento se encuentra muy por debajo de los 250 kg ha-1 año-1cacao seco, que es el rendimiento del cultivo en el país (Catie-PCC, 2010). 
El resultado de la revisión bibliográfica permitió determinar que la poca productividad de las áreas de cacao y la poca cultura de asociatividad entre los productores de cacao de la región, están limitando a las organizaciones a beneficiar y comercializar mayores volúmenes de cacao y, frenando el crecimiento de las organizaciones tanto en el número de socios como como en las áreas de cacao.

\section{Limitantes y ventajas comparativas de las organizaciones}

La variable de cadena de valor está compuesta por nueve indicadores. Cinco indicadores corresponden a la dimensión de actividades primarias y cuatro a la dimensión de actividades de apoyo. Los cinco indicadores de actividades primarias a su vez se dividen en: logística de entrada, operaciones o producción, logística de salida, mercadotecnia, ventas y servicio posventa.

Los cuatro indicadores de actividades de apoyo a su vez se dividen en: adquisición, desarrollo tecnológico, administración de recursos humanos e infraestructura organizacional.

\section{Limitantes. Actividades primarias}

Al obtener el valor de los subíndices de los indicadores de la variable cadena de valor (ver Figura 2 y 3), se observa que los subíndices que muestran un valor menor a 0.5 son aquellos aspectos de la cadena de valor a los cuales se deben poner mayor atención, no sin antes tener en cuenta que inclusive los subíndices con valores menores a o.70 permiten una adecuada cadena de valor y que también deben ser coordinados y optimizados; por consiguiente, se tienen las siguientes limitantes:

- Las organizaciones no cuentan con lugares específicos para resguardar sus instrumentos y equipos de trabajo.

- Inadecuada frecuencia de monitoreo durante el proceso de fermentación y secado del grano.

- Tanto las actividades de mantenimiento de los equipos de fermento y secado, como la frecuencia con que se realizaron durante el 2014, están mal realizadas.

- No hay planificación de las actividades de acopio, beneficiado y comercialización del producto.

- Pocas organizaciones han tratado de buscar nuevos compradores.

- No se está realizando ajustes a la calidad del producto producido. 


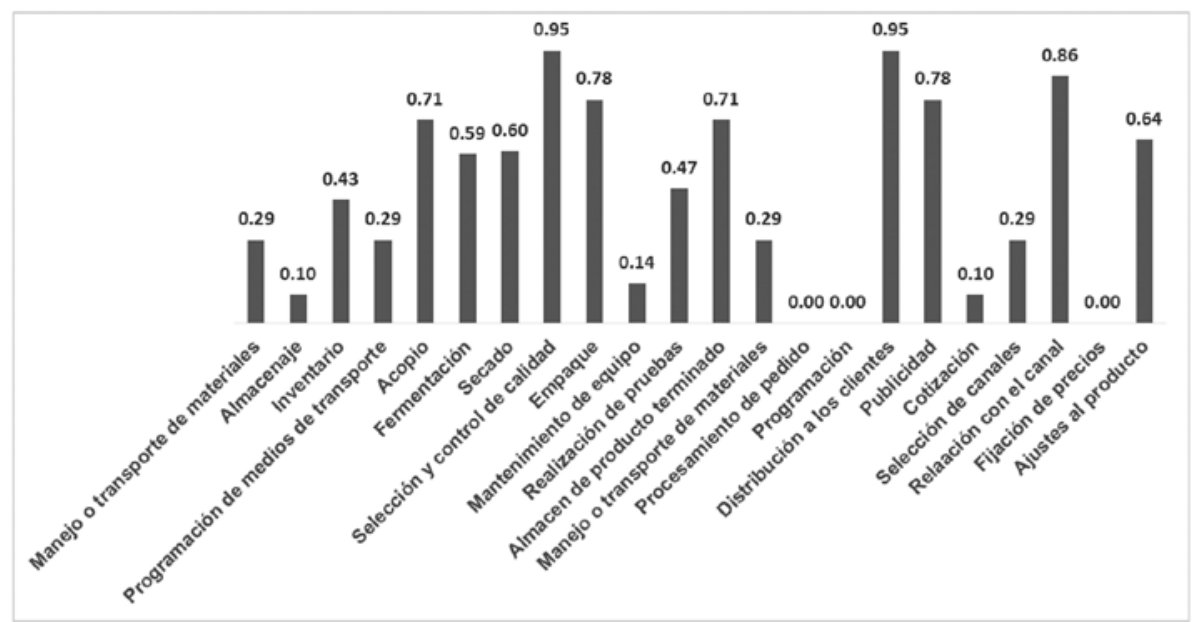

Figura 1: Valores de los subindicadores de las actividades primarias de la cadena de valor en una escala de o a1.

Fuente: Elaboración propia con datos de encuestas.

\section{Limitantes. Actividades de apoyo}

1. Falta de calidad en la materia prima adquirida para el beneficiado de cacao (80\%).

2. Poco uso de equipos tecnológicos durante el proceso de beneficiado del cacao, en su mayoría los responsables del beneficiado del cacao están aplicando procedimientos empíricos.

3. Poco uso de tecnologías integradas al proceso de beneficiado de cacao, que permitirían acelerar y estandarizar el proceso de secado del grano.

4. Falta de elaboración y ejecución de planes de capacitación tanto para el personal del área administrativa como para el personal que labora en el beneficiado de cacao.

5. Falta de planeación y supervisión de todas y cada una de las actividades para el beneficiado y comercialización del grano de cacao, por parte de los consejos de dirección de cada organización. 


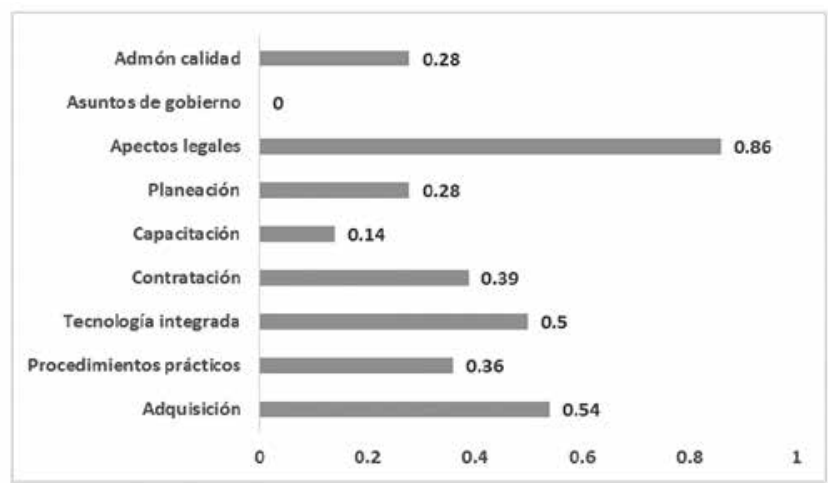

Figura 2: Valores de los subindicadores de las actividades de apoyo de la cadena de valor en una escala de o a1.

Fuente: Elaboración propia con datos de encuestas.

Al comparar los indicadores de la cadena de valor, se determinó que los indicadores; logística de entrada, logística de salida, mercadotecnia y ventas, adquisición, administración de recursos humanos, desarrollo tecnológico e infraestructura organizacional, están incidiendo negativamente en la calidad del producto obtenido y por ende frenando la competitividad de las organizaciones, por lo que se les debe poner especial atención para mejorar la situación de las organizaciones, ver Figura 4.

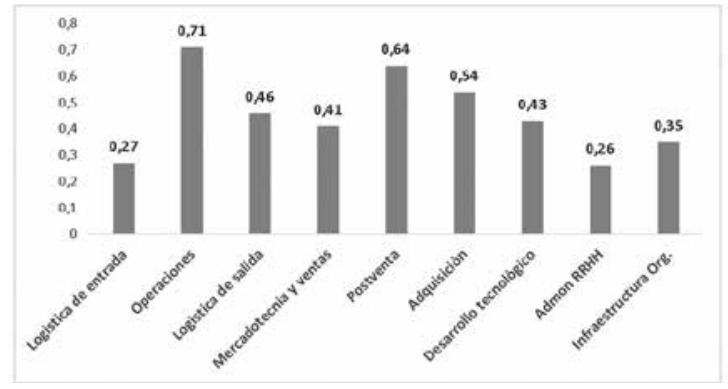

Figura 3: Valores de los indicadores de la cadena de valor en una escala de o a 1.

Fuente: Elaboración propia con datos de encuestas

\section{Ventajas comparativas}

El resultado del análisis de la cadena de valor determinó las siguientes ventajas comparativas entre organizaciones:

- Disponibilidad de áreas productivas de cacao.

- Disponibilidad de infraestructura (centros de acopio).

- Producción con buen porcentaje de calidad. 
- Uso de diversas tecnologías en el proceso de beneficiado.

De manera general se puede decir que dos organizaciones de las 7 en estudio son las que presentan más ventajas comparativas; tienen las mayores áreas de producción, mayores volúmenes de acopio, beneficiado y comercialización de cacao con calidad, lo que les permitió disminuir sus costos y obtener una ventaja competitiva. Estos resultados coinciden con los enunciados de Porter (2006) quien señala que una ventaja competitiva se logra cuando las actividades de la cadena de valor se realizan mejor.

Tabla 1: Ventajas comparativas entre las organizaciones.

\begin{tabular}{|c|c|c|c|c|c|c|c|c|}
\hline $\begin{array}{c}\text { Organización } \\
\text { Indicador }\end{array}$ & Coos & Coop & Cood & Asih & UCA & SS & Comp & Total \\
\hline \multicolumn{9}{|l|}{ Operación } \\
\hline Acopio $^{\mathrm{TM}}$ & 92.35 & 77.98 & 21,98 & 3.0 & 23.26 & 8.19 & 27.41 & 254.17 \\
\hline Centros de acopio & 4 & 4 & 1 & 3 & 1 & 4 & 1 & 18 \\
\hline \multicolumn{9}{|l|}{ Calidad del producto } \\
\hline$\% A$ & 25 & 24.37 & 0 & 0 & $\mathrm{O}$ & 0 & 17 & \\
\hline$\% B$ & 38.1 & 46.94 & 40.2 & 80 & 56.25 & 60 & 65.7 & \\
\hline$\% C$ & 36.9 & 28.69 & 59.8 & 20 & 43.75 & 40 & 17.3 & \\
\hline \multicolumn{9}{|l|}{ Adquisición } \\
\hline $\begin{array}{l}\text { Productores socios y } \\
\text { no socios }\end{array}$ & 173 & 191 & 36 & 45 & 186 & 106 & 234 & 971 \\
\hline Áreas de cacao & 255.58 & 156.06 & 82.45 & 58.25 & 218.31 & 118.66 & 16.71 & 906.03 \\
\hline \multicolumn{9}{|l|}{ Operación } \\
\hline Acopio $^{\mathrm{TM}}$ & 92.35 & 77.98 & 21,98 & 3.0 & 23.26 & 8.19 & 27.41 & 254.17 \\
\hline Centros de acopio & 4 & 4 & 1 & 3 & 1 & 4 & 1 & 18 \\
\hline \multicolumn{9}{|l|}{ Calidad del producto } \\
\hline$\% \mathrm{~A}$ & 25 & 24.37 & 0 & 0 & 0 & 0 & 17 & \\
\hline$\% B$ & 38.1 & 46.94 & 40.2 & 80 & 56.25 & 60 & 65.7 & \\
\hline$\% C$ & 36.9 & 28.69 & 59.8 & 20 & 43.75 & 40 & 17.3 & \\
\hline \multicolumn{9}{|l|}{ Desarrollo tecnológico } \\
\hline Cajillas malla acerada & $x$ & $x$ & $x$ & & & & & \\
\hline Horno Samoa & $x$ & $x$ & $x$ & $x$ & & & & \\
\hline
\end{tabular}

Coos: Coosemucrim; Coop: Cooprocafuc; Cood: Coodeprosa; Asih: Asiherca; SS: Sano y Salvo; Comp: Compor Fuente: Elaboración propia con datos de encuestas 


\section{Competitividad en las organizaciones cacaoteras}

$\mathrm{Al}$ obtener el valor de los indicadores de competitividad en una escala de o a 1, los valores iguales o menor a .05 son a los que se debe procurar mayor atención. En ese sentido, las organizaciones deben poner especial atención a; Calidad, Productividad, Cuota de mercado, Costos y Permanencia en el mercado; para mejorar su competitividad. (Ver Figura 5).

Con los resultados del valor de los indicadores dos organizaciones apuntan a ser competitivas son, sin embargo, deben mejorar los indicadores antes mencionados. (Ver Figura 6).

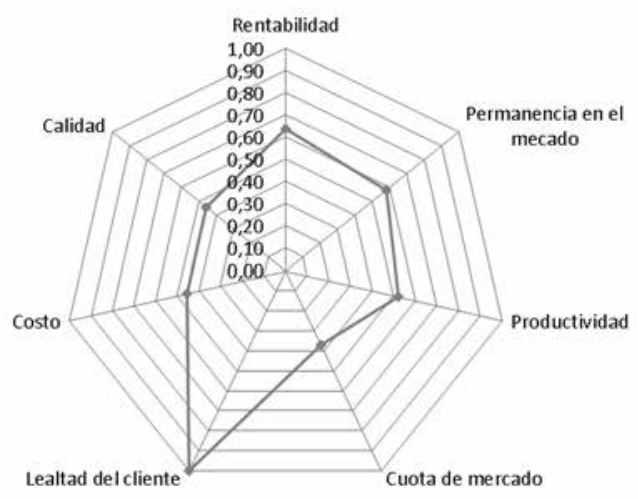

Figura 4: Valor de los indicadores de competitividad.

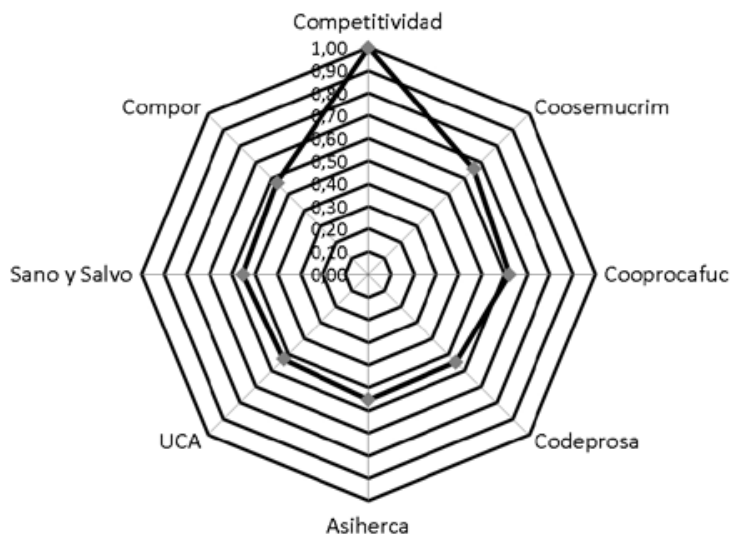

Figura 5: Situación de las organizaciones de acuerdos a los indicadores de competitividad. 


\section{Comprobación de las hipótesis}

Hi: La cadena de valor influye en la competitividad

En el análisis de relación entre las actividades que integran la cadena de valor y la competitividad se encontró una "correlación positiva considerable" $r=0.7162$, lo cual de acuerdo a Hernández (2010), indica que la variable cadena de valor se relaciona positivamente en un $71.62 \%$, y contribuye directamente en la competitividad. Por tanto, la hipótesis de trabajo es aceptada porque la variable cadena de valor explica en un $51.29 \%$ a la variable competitividad, ver figura 7 .

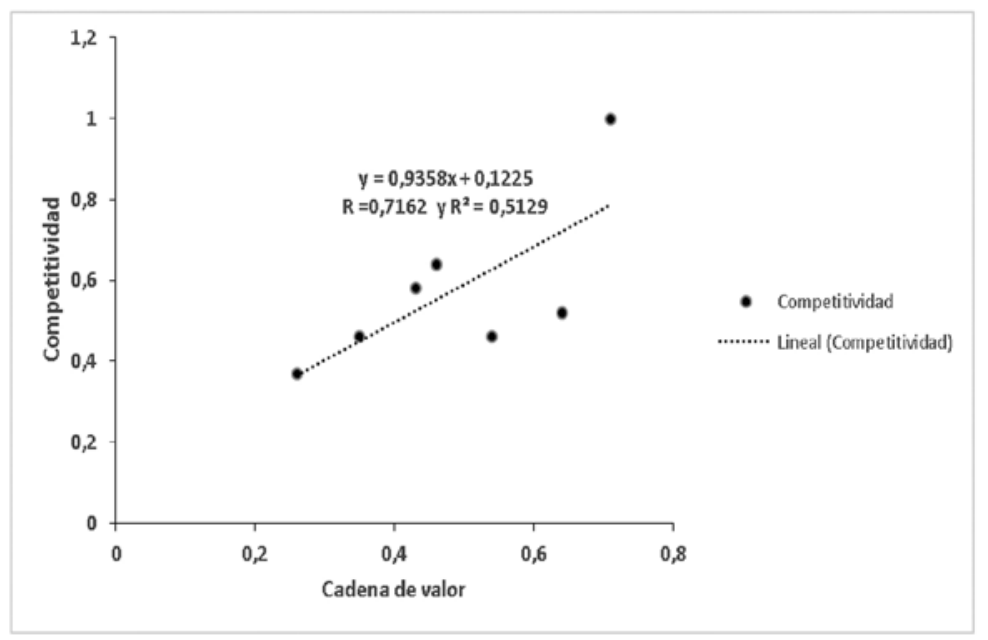

Figura 6: Diagrama de dispersión que muestra la relación entre cadena de valor y competitividad. Fuente: Elaboración propia con datos de la encuesta.

\section{Conclusiones}

- Las actividades primarias no están contribuyendo a incrementar el valor del producto que las organizaciones llevan al mercado. Siendo el indicador de operaciones o producción el más débil.

- Las actividades de procesamiento no están siendo apoyadas por los recursos; Infraestructura, recursos humanos, desarrollo tecnológico y abastecimiento de materia prima. Esto debido a la falta de recursos en algunas organizaciones y a la falta de planificación en otras.

- Las limitantes que se evidenciaron al estudiar la cadena de valor, se dieron en el proceso productivo, siendo las principales la calidad del producto; los bajos volúmenes comercializados y la falta de integración de tecnologías al proceso productivo. 
- Dos organizaciones presentaron ventajas comparativas, las que les permitió minimizar los costos y posicionarse en una mejor situación competitiva, en comparación a las otras organizaciones en estudio.

- De acuerdo a la correlación de Pearson efectuada se comprobó la incidencia de la cadena de valor para obtener niveles de competitividad adecuados.

- De acuerdo a los resultados del análisis de la variable competitividad, ninguna de las organizaciones estudiadas es competitiva, debido a que son organizaciones que presentaron bajos niveles de desempeño en cinco indicadores considerados en este estudio: permanencia en el mercado del cacao, productividad, cuota de mercado, costos y calidad del producto.

\section{Lista de referencias}

Catie-PCC. (2010). Cadena Productiva de Cacao de Nicaragua. Informe Final de Consultoría. $27 \mathrm{p}$.

Grant, R. M. (1996). Dirección estratégica: conceptos, técnicas y aplicaciones. Madrid: Civitas Ediciones.

Guitart, L. (2005). La ruptura de la cadena de valor como consecuencia de la subcontratación: una investigación cualitativa mediante el estudio de casos. Barcelona: Universidad de Barcelona.

Hernández, E. (200o). La competitividad industrial en México. México: UAM; Plaza y Valdés.

Manso, F. J. (2003). Diccionario enciclopédico de estrategia empresarial. Madrid, España: Ediciones Días de Santos.

Müller, G. (1995). El caleidoscopio de la competitividad. Revista de la CEPAL, número $56,137-148$.

Porter, M. (2006). Ventaja competitiva. 5ta. reimpresión. España: Editorial CECSA.

--- (1987). Ventaja competitiva. México: CECSA.

--- (1985). Competitive Advantage. Nueva York: Free Press

Ruesga, S. M. \& Da Silva B. J. (2007). Competitividad y globalización: Nuevos y viejos desafíos. Papeles del Este, número 14, 1-27. 